\title{
La inversión en la educación en Madre de Dios *
}

\section{The investment in the education in Madre de Dios}

\author{
Rolando Alberto Molina Bocangel ${ }^{* *}$
}

\section{RESUMEN}

La presente investigación busca determinar el impacto de los actuales niveles de inversión destinados a la educación en la formación de capital humano que sustente un crecimiento y desarrollo sostenible en el departamento de Madre de Dios. El tipo de investigación es descriptivo, explicativo y correlacional.

Como conclusión se determinó que los mejores niveles de inversión destinados a la educación son importantes para la formación de capital humano que sustente un crecimiento y desarrollo sostenible.

Palabras clave: Inversión; educación; capital humano.

JEL: I21, I25, I28, 015, 021

\footnotetext{
* En el marco del desarrollo de mis estudios de maestría en Desarrollo Empresarial y Regional en la Universidad Mayor de San Marcos, se decidió plantear la importancia de la Inversión en educación para el desarrollo del capital humano en Madre de Dios.

** Economista, Universidad Nacional San Antonio Abad, Cusco, Perú, estudios de magister en Economía mención Desarrollo Empresarial y Regional, Universidad Nacional Mayor de San Marcos, Lima, Perú, Sub-gerente de Programas Sociales y Proyectos de la Gerencia de Desarrollo Social del Gobierno Regional de Madre de Dios, Avenida La Joya L-3, Puerto Maldonado, Madre de Dios.

E-mail: almolbo@hotmail.com

(C) Los autores. Este artículo es publicado por Pensamiento Crítico de la Facultad de Ciencias Económicas, Universidad Nacional Mayor de San Marcos. Este es un artículo de acceso abierto, distribuido bajo los términos de la licencia Creative Commons Atribucion - No Comercia_Compartir Igual 4.0 Internacional. (http://creativecommons.org/licenses/by-nc-sa/4.0/) que permite el uso no comercial, distribución y reproducción en cualquier medio, siempre que la obra original sea debidamente citada.
} 


\section{ABSTRACT}

The present research seeks to determine the impact of the current levels of investment destined to education in the formation of human capital that sustain growth and economic development in Madre de Dios. The type of research is descriptive, explanatory and correlational.

In conclusion, it was determined that the best levels of investment destined to education are important for the formation of human capital that supports growth and economic development.

Keywords: Investment; education; human capital.

JEL: I21, I25, I28, 015, 021

\section{Introducción}

Uno de los indicadores más difundidos para medir la riqueza de una nación o región de un país (grado de desarrollo alcanzado), es sin lugar a dudas en términos socio- económicos, el producto bruto interno per- cápita. En ese marco, hoy en día existen países y regiones cuyos habitantes cuentan con ingresos superiores a los $\$ 30,000^{1}$, frente a otros cuyos habitantes sobreviven con ingresos menores a $\$ 1^{2}$ al día.

Diferentes autores señalan que la educación es un factor clave para reducir la brecha entre ricos y pobres, y de esta manera romper con el círculo vicioso en que éstos últimos se hallan inmersos. Al respecto cabe indicar que, la formación de capital humano, a través de la educación, ofrece mayores oportunidades a quien puede acceder a ella, que se traduce en mayor libertad entre otros aspectos.

La inversión en educación contribuye de manera significativa con el crecimiento y desarrollo económico de las regiones, al generar elevadas tasas de rentabilidad social y de retorno privado. De los tres niveles educativos, la educación básica primaria genera la mayor contribución al crecimiento y desarrollo económico. En efecto, un estudio del Banco Mundial sobre la educación en América Latina y el Caribe calculó la tasa de

1 Estados Unidos, Dinamarca, Guinea Ecuatorial, Irlanda, Luxemburgo, Noruega, Suiza, según datos del PNUD 2004.

2 Bangladesh, Burundi, Camboya, Gambia, El Salvador, India, Nicaragua, entre otros. Mas de un 30\% de la población se encuentra en esta situación, según PNUD - 2004. 
rentabilidad social de la educación básica primaria en 17,9\%, por encima de las tasas de secundaria $(12,8 \%)$ y educación superior $(12,3 \%)$. Por su parte, la tasa de retorno privado en básica primaria $(26,2 \%)$ también fue superior a las tasas de la educación secundaria y universitaria ${ }^{3}$.

Adicional a lo anterior, diversos estudios empíricos han constatado que "los grupos de menores ingresos reciben la mayor parte del gasto público, especialmente en primaria. En el caso de la educación superior, la distribución es regresiva, pues el gasto se concentra en los quintiles de mayores ingresos" 4 .

En este sentido, las tasas de retorno son decrecientes en la medida que aumenta el nivel educativo.

En base a lo mencionado anteriormente, podemos decir que, la formación de Capital Humano $(\mathrm{CH})$, representa el indicador más confiable del crecimiento y desarrollo económico de una región y de un país, el determinante fundamental del avance tecnológico en todos los sectores económicos y responsable en gran medida, de aquellas regiones y países que se enrumban a la senda del desarrollo económico.

Es preciso señalar que existen variados antecedentes bibliográficos sobre el tema objeto de estudio entre los cuales podemos mencionar por ejemplo, el Informe que sobre Capital Humano en Chile realizo José Joaquín Brunner Y Gregory Elacqua con apoyo de la Escuela de Gobierno, de la Universidad ADOLFO IBAÑEZ. Así mismo podemos mencionar también a Jhonson, H.G, de México con "Hacia un enfoque del desarrollo económico partiendo de un concepto generalizado de la acumulación de capital" entre otros.

En el caso especifico del Perú y de Madre de Dios, si bien es cierto se han invertido recursos económicos en el desarrollo del capital humano, los resultados a pesar de ser favorables, no cumplen aun con un mínimo necesario para las exigencias del país, además están muy por debajo de los resultados que se hubiesen podido lograr con las sumas invertidas.

3 Cohen, Ernesto, “Hacia dónde va el gasto público en educación? Logros y desafíos”, Vol. I, Serie Políticas Sociales, 42, CEPAL, Santiago de Chile, 2000.

4 Corpoeducación, Situación de la educación básica, media y superior en Colombia, Editorial El Tiempo, Fundación Antonio Restrepo Barco, Bogotá, 2001. 
La inversión en educación registrada en el Perú, como porcentaje del PBI $(2.5 \%)^{5}$ se encuentra por debajo del promedio registrado por otros países latinoamericanos é industrializados y en el caso del departamento de Madre de Dios.

Es importante señalar de otro lado que una población dotada de niveles adecuados de capacitación educativa constituye el ingrediente primordial para incrementar el capital humano en el departamento de Madre de Dios y el Perú, una población sin educación, no ofrece el mejor insumo para la formación de un sólido stock de capital humano, que permitiría fomentar el crecimiento sostenido de una economía.

De este modo, el objetivo fundamental del presente trabajo es determinar el impacto de los actuales niveles de inversión destinados a la educación, en la formación de capital humano que sustenten un crecimiento y desarrollo económico sostenible en Madre de Dios.

\section{A. Situación Problemática}

Madre de Dios contaba con una población calculada al año 2012 de 127,639 habitantes (Ver Cuadro $\mathrm{N}^{\circ}$ 01) con una tasa de crecimiento de $3.9 \%$, una densidad poblacional de 1.53 hab./km2 y tenía los mayores niveles de migración provenientes de la sierra sur. El 73\% de la población habitaba en el área urbana.

La población joven era mayoritaria, más del 31.2\% era menor de 15 años y el 54\% estaba por debajo de los 25 años. La población en edad de trabajar (de 15 a 64 años) representaba el $66.3 \%$ del total y las personas de la tercera edad llegaban al 2.5\%.

En Madre de Dios el 12.7\% vivía en pobreza y el 1.8\% en extrema pobreza ${ }^{6}$. Además, las familias tenían al menos una necesidad básica insatisfecha conformando el 29.4\% de la población.

Por los pocos ingresos del departamento, el 12.7\% de los hogares accedían a un programa de asistencia social.

5 Inversión en la calidad de la educación publica en Perú y su efecto sobre la fuerza de trabajo y pobreza. GRADE 2002 - 2004.

6 Comprendió estadístico Sociodemográfico del 2012. 
Cuadro $\mathbf{N}^{\circ}$ 01. Población de Madre de Dios

\begin{tabular}{|c|c|}
\hline TOTAL & 127,639 \\
\hline TAMBОРАТА & 91,988 \\
\hline Tambopata & 71,774 \\
\hline Inambari & 9,415 \\
\hline Las Piedras & 5,779 \\
\hline Laberinto & 5,040 \\
\hline MANU & 22,906 \\
\hline Manu & 2,974 \\
\hline Fitzcarrald & 1,446 \\
\hline Madre de Dios & 11,596 \\
\hline Huepetuhe & 6,890 \\
\hline TAHUAMANU & 12,745 \\
\hline Iñapari & 1,458 \\
\hline Iberia & 8,081 \\
\hline Tahuamanu & 3,196 \\
\hline
\end{tabular}

Fuente: INEI

Madre de Dios contribuyó con menos del 1\% al PBI del Perú y la contribución de la actividad económica agraria al PBI departamental fue de 9.2\% y de la minería 37.9\%.

En el país la educación ha logrado avances en la atención en primaria. Existen atrasos en cuanto a la calidad educativa, pues una buena cantidad de estudiantes no llegan a las metas de aprendizaje programados.

La Evaluación Censal de Estudiantes del Ministerio de Educación, muestra incrementos en las metas de aprendizaje, pero los avances son aún mínimos. En Perú, el 2013 las/los estudiantes de segundo grado de primaria el $33.0 \%$ y el $16.8 \%$ lograron las metas de aprendizaje en Comprensión Lectora y en Comprensión Matemática.

En Madre de Dios, los estudiantes alcanzaron el $17.7 \%$ y el $5.4 \%$ de las metas de aprendizaje en Comprensión Lectora y en Comprensión Matemática. 
Cuadro $\mathbf{N}^{\circ}$ 02. Calidad Educativa

\begin{tabular}{llcccccc} 
Indicador & Territorio & $\mathbf{2 0 0 8}$ & $\mathbf{2 0 0 9}$ & $\mathbf{2 0 1 0}$ & $\mathbf{2 0 1 1}$ & $\mathbf{2 0 1 2}$ & $\mathbf{2 0 1 3}$ \\
\hline $\begin{array}{l}\text { Niños y niñas que alcanzan sufi- } \\
\text { ciencia en el logro de las compe- }\end{array}$ & Perú & 16.9 & 23.1 & 28.7 & 29.8 & 30.9 & 33.0 \\
$\begin{array}{l}\text { tencias de comprensión lectora en } \\
\text { segundo grado (\%) }\end{array}$ & $\begin{array}{l}\text { Madre de } \\
\text { Dios }\end{array}$ & 10.4 & 12.4 & 16.3 & 17.2 & 19.6 & 17.7 \\
$\begin{array}{l}\text { Niñas y niños que alcanzan sufi- } \\
\text { ciencia en el logro de las competen- } \\
\text { cias de comprensión matemática } \\
\text { en segundo grado (\%) }\end{array}$ & $\begin{array}{l}\text { Perú } \\
\text { Madre de }\end{array}$ & 9.4 & 13.5 & 13.8 & 13.2 & 12.8 & 16.8 \\
\hline
\end{tabular}

Fuente: Ministerio de Educación, Secretaría de Planificación Estratégica, Oficina de Seguimiento y Evaluación Estratégica, Unidad de Estadística, 2014

\section{B. Inversión en educación}

El cuadro $\mathrm{N}^{\circ} 03$ muestra el comportamiento de la inversión pública en educación como componente de la inversión pública, a nivel país ha seguido una media de 13.1\% para el periodo 2004-2012. Madre de Dios en este periodo tuvo una media de $19.88 \%$ y un ciclo más variado, excepto 2009 y 2010 en que estuvo menos del promedio nacional.

Cuadro $\mathbf{N}^{\circ}$ 03. Inversión Pública en Educación como Componente de la Inversión Pública

\begin{tabular}{|c|c|c|c|c|c|c|c|c|c|c|}
\hline Indicador & & 2004 & 2005 & 2006 & 2007 & 2008 & 2009 & 2010 & 2011 & 2012 \\
\hline $\begin{array}{l}\text { Inversión } \\
\text { pública en } \\
\text { educación }\end{array}$ & Perú & 15.6 & 16.2 & 15.7 & 14.7 & 14.3 & 14.3 & 14.3 & 13.6 & 13.5 \\
\hline $\begin{array}{l}\text { ponente de } \\
\text { la Inversión } \\
\text { pública }\end{array}$ & $\begin{array}{l}\text { Madre } \\
\text { de Dios }\end{array}$ & 32.3 & 37.4 & 20.1 & 21.3 & 19.0 & 6.6 & 11.5 & 16.6 & 14.1 \\
\hline
\end{tabular}

Fuente: Ministerio de Educación, Secretaría de Planificación Estratégica, Oficina de Seguimiento y Evaluación Estratégica, Unidad de Estadística, 2013

Con respecto a la educación ocupacional, en la ciudad de Puerto Maldonado hay solo dos centros que la imparten, uno de gestión estatal y el otro de gestión privada.

En educación superior no universitaria existen el Instituto Superior Pedagógico Nuestra Señora del Rosario y el Instituto Superior Tecnológico Jorge Basadre Grohmann ubicados en la ciudad de Puerto Maldonado de la Provincia de Tambopata, el Instituto Superior del Manu en la pro- 
vincia del mismo nombre ubicado en la villa de Salvación y el Instituto Superior Tecnológico de Iberia, ubicado en la ciudad del mismo nombre de la provincia de Tahuamanu. El principal centro de estudios superiores del departamento es la Universidad Nacional Amazónica de Madre de Dios (UNAMAD), a la que se agregan las filiales de la Universidad Particular Andina del Cusco, de la Universidad San Antonio Abad del Cusco y de la Universidad Alas Peruanas.

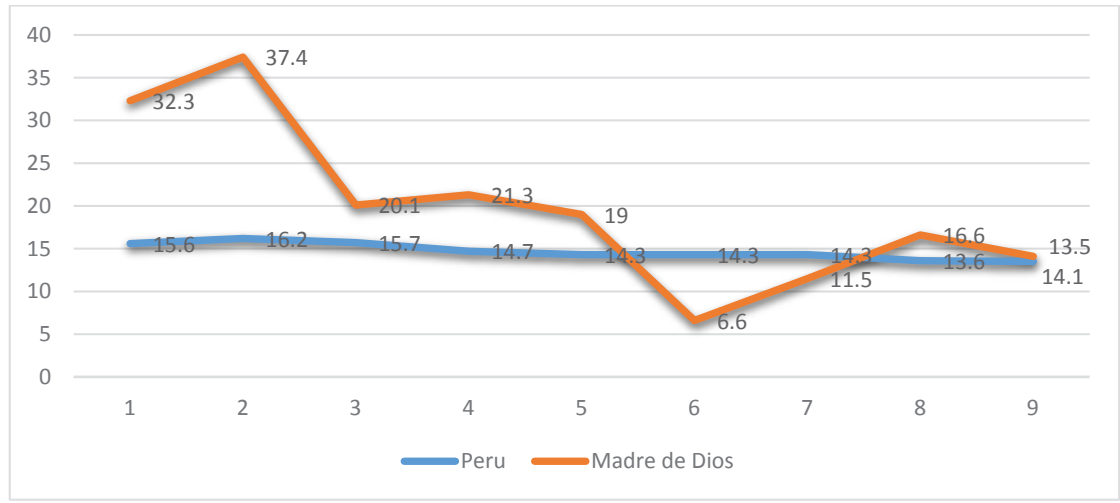

Gráfico $N^{\circ}$ 01. Inversión Pública en Educación como Componente de la Inversión Pública Fuente: Ministerio de Educación, Secretaría de Planificación Estratégica, Oficina de Seguimiento y Evaluación Estratégica, Unidad de Estadística, 2013.

Cuadro $\mathbf{N}^{\circ}$ 04. Cantidad de Alumnos Matriculados Semestre 2012 - II por Carrera

\begin{tabular}{llcc} 
№ & CARRERA & CODIGO DE CARRERA & CANT. \\
1 & ING.AGROIDUSTRIAL & 010 & 202 \\
2 & ING. FORESTAL Y MEDIO AMBIENTE & 020 & 322 \\
3 & ING. DE SISTEMAS E INFORMÁTICA & 021 & 151 \\
4 & MEDICINA VETERINARIA Y ZOOTECNIA & 051 & 116 \\
5 & ECOTURISMO & 032 & 265 \\
6 & CONTABILIDAD Y FINANZAS & 037 & 207 \\
7 & ADMINISTRACION YNEGOCIOS INTERNACIONALES & 036 & 200 \\
8 & EDUCACION MATEMATICA YCOMPUACIÓN & 031 & 59 \\
9 & EDUCACION INICIALY ESPECIAL & 029 & 60 \\
10 & EDUCACION PRIMARIA E INFORMÁTICA & 030 & 35 \\
11 & DERECHOY CIENCIAS POLÍTICAS & 041 & 202 \\
12 & ENFERMERIA & 061 & 105 \\
\hline & & TOTAL & $\mathbf{1 9 2 4}$ \\
\hline
\end{tabular}

Fuente: Universidad Nacional Amazónica de Madre de Dios. 
Toda esta situación mencionada anteriormente influye en la existencia de un capital humano poco competitivo, que no aporta al desarrollo económico y social del departamento, no obstante tener una tasa de analfabetismo reducida, lo cual refleja que dadas las necesidades, los pobladores no continúan su educación, ya sea por falta de recursos o porque no existe la oferta educativa adecuada, para el desarrollo del departamento.

Como se puede apreciar todo es un círculo vicioso, si no existe educación, no existe capital humano competitivo y a la vez no existe desarrollo económico y social en la región.

El crecimiento y desarrollo del departamento de Madre de Dios está ligado con la formación de capital humano, y éste a la vez depende en gran medida de la educación que se brinda en los niveles de inicial, primaria, secundaria y superior.

\section{Marco Teórico}

\section{a. La Educación}

\section{i. Aspectos Generales}

El camino hacia el desarrollo de un país pasa necesariamente por la Educación. Un país exitoso es el que tiene gente inteligente porque invierte en mejor educación (...) las personas e ideas brillantes atraen más empresas al país, y un gobierno inteligente garantiza las condiciones idóneas para que las compañías inviertan en sus trabajadores y en su territorio ${ }^{7}$. Mas inversión e inversión de calidad, supondrá mejor desarrollo humano y desarrollo educativo, menos analfabetismo, mas escolaridad y mejores oportunidades lo que deberá impactar en el aparato empresarial y productivo.

Países que invierten más en educación obtienen mejores lugares de desempeño competitivo y productivo. En ese sentido, es probable que se necesite incluir nuevos ingredientes de corte empresarial, tales como largo plazo, visión estratégica, liderazgo, costo beneficio, tasa de retorno, evaluación objetiva, rendición de cuentas y sobre

7 ¿Altruismo o necesidad? Revista Estrategia \& Negocios N. 107 . Meses de Noviembre y Diciembre 2008. Pág. 184.. 
todo meritocracia. Más aún en sistemas educativos latinoamericanos, que están montados sobre dos pilares; una densa arquitectura burocrática y un inexpugnable marco legal, factores que limitan las respuestas a los retos de la sociedad del conocimiento.

\section{ii. Definición}

La educación para DURKHEIM: "es un proceso de transmisión cultural de una generación a otra, de las generaciones adultas a las generaciones jóvenes, es un proceso social lento por su origen como por sus funciones". Varia en el tiempo y adopta formas distintas en el espacio reflejando siempre toda la escala de valores de la sociedad, posibilita el crecimiento del ser humano en los ámbitos de la vida permitiéndole desenvolverse en medio de su contexto de forma adecuada.

\section{iii. Principios educacionales}

El sistema educativo peruano, debe tener principios enunciados en el Plan de Corto, Mediano y Largo Plazo del Ministerio de Educación, y que serian los siguientes:

La Ética promotora de los valores, solidaridad, libertad, honestidad y responsabilidad.

La Equidad en las oportunidades de acceso, permanencia y trato.

La Inclusión de grupos sociales vulnerables.

La Democracia, respeto a los derechos humanos, la libertad de conciencia, pensamiento y opinión.

La inter-culturalidad y el respeto y reconocimiento a las diferencias culturales.

La creatividad e innovación para el Desarrollo Humano.

\section{iv. Demanda de la Educación}

Son las aspiraciones, deseos y necesidades de la población. A nivel social, basada en la idea que la educación es un Bien y Servicio para la población. Es para satisfacer necesidades sociales prioritarias y es una inversión. 
La demanda de la persona está fundada en los derechos humanos y en el interés de formarse, para mejorar, invertir en su desarrollo tiene a la educación como inversión.

La Educación debe atender tanto la demanda interna de la población peruana y mater deitana, y la demanda de carácter externo, proveniente de Latinoamérica y el Mundo.

\section{Demanda de Carácter Externo}

Alineada con el avance de la ciencia y la tecnologia, la educación debe preparar a los niños y jóvenes para que desarrollen en una sociedad competitiva y asumir los aportes del exterior que beneficien al país y la región. Es necesario que la educación promueva el desarrollo de capacidades para que los niños, las niñas, las/los adolescentes y las/los jóvenes, manejen con eficiencia y eficacia información que incremente su habilidad para la captación de conocimientos.

Concordante con la Mundialización, cuya característica es la universalización de patrones de vida y de consumo de los países desarrollados, que vienen a ser referentes para la mayoría de la población del mundo.

La educación debe ser la base en la formación de un razonamiento analítico y crítico para la asimilación selectiva de los modelos de comportamiento que se difunden a través de los medios de comunicación, orientada a la formación de capacidades para decidir sobre la construcción de programas y proyectos propios, individual y colectivo de las/los jóvenes.

\section{Demanda de Carácter Interno}

La población peruana se halla comprometida con la construcción de formas superiores de existencia, donde se hayan superado los problemas que aquejan a grandes sectores de nuestra población y principalmente los que propician la baja autoestima, la pérdida de la identidad, la discriminación, la pauperización, el desaliento. Frente a ello, el país demanda una educación signada por valores, enraizada en nuestra realidad, orientada al mejoramiento de la calidad de vida y al desarrollo del país. En particular, la población demanda de la Educación una atención prioritaria a las 
necesidades de los niños y las niñas en proceso de crecimiento e inserción en la vida familiar y comunitaria.

\section{La educación y el desarrollo humano en Madre de Dios}

\section{A. Análisis de la Educación}

$\mathrm{Al}$ año 2012 Madre de Dios, muestra los indicadores siguientes:

El 3.6\% de la población mayor de 15 años es analfabeto, indicador por debajo del promedio nacional y el número de años de estudio promedio de la población mayor de 15 años es de 9.8 años, indicador también por debajo del promedio nacional según el Ministerio de Educación.

Cuadro $\mathbf{N}^{\circ}$ 05. Número de alumnos en el Sistema Educativo, Madre de Dios 2012

\begin{tabular}{|c|c|c|c|c|c|c|c|}
\hline \multirow{2}{*}{$\mathrm{N}^{\circ}$} & \multirow{2}{*}{ NIVEL Y MODALIDAD } & \multicolumn{5}{|c|}{ UNIDADES DE GESTION EDUCATIVA } & \multirow{2}{*}{$\begin{array}{c}\text { TOTAL } \\
\text { PUBLICO }\end{array}$} \\
\hline & & TAMBOPATA & MANU & TAHUAMANU & LA CONVENCION & PURUS & \\
\hline \multicolumn{8}{|c|}{ A. PROGRAMA ESCOLARIZADO } \\
\hline \begin{tabular}{|r|}
1 \\
\end{tabular} & EDUC. INICIAL JARDIN & 4204 & 667 & 409 & 295 & 192 & 5767 \\
\hline 2 & EDUC. CUNA JARDIN & 384 & & & & & 384 \\
\hline 3 & EDUC. PRIMARIA MENORES & 12786 & 2194 & 1227 & 1502 & 765 & 18474 \\
\hline 4 & CEBA & 1014 & 94 & 89 & & & 1197 \\
\hline 5 & EDUC. SEC. MENORES & 8395 & 1277 & 829 & 484 & 486 & 11471 \\
\hline 6 & EDUC. SEC. ADULTOS & & & & & & \\
\hline 7 & EDUC. ESPECIAL & 62 & & & & & 62 \\
\hline 8 & EDUC. OCUPACIONAL & 278 & & & 23 & & 301 \\
\hline 9 & EDUC. MAGISTERIAL & 51 & & & & & 51 \\
\hline 10 & EDUC. SUP. TECNOLOGICO & 329 & 51 & 142 & & & 522 \\
\hline & SUB TOTAL & 27503 & 4283 & 2696 & 2304 & 1443 & 38229 \\
\hline \multicolumn{8}{|c|}{ B. PROGRAMA NO ESCOLARIZADO } \\
\hline \begin{tabular}{|r|}
1 \\
\end{tabular} & PRONOEI & 631 & 55 & 47 & 56 & 0 & 789 \\
\hline \multicolumn{8}{|c|}{\begin{tabular}{l|l}
2 & EDUC. SEC. ADULTOS \\
\end{tabular}} \\
\hline & SUB TOTAL & 631 & 55 & 47 & 56 & 0 & 789 \\
\hline & TOTAL (A+B) & 28134 & 4338 & 2743 & 2360 & 1443 & 39018 \\
\hline
\end{tabular}

El 94.9\% de la población total entre 6 a 11 años asiste a educación primaria, indicador superior al promedio nacional, el $76.6 \%$ de la población total entre 12 a 16 años asiste a educación secundaria, indicador que también es superior al promedio nacional.

\section{Acceso educativo}

Los más altos indicadores, se muestran en primaria y secundaria a nivel nacional como regional. 
Cuadro $\mathbf{N}^{\circ}$ 06. Acceso por Nivel Educativo

\begin{tabular}{|c|c|c|c|c|c|c|c|}
\hline \multicolumn{2}{|l|}{ Indicador } & 2007 & 2008 & 2009 & 2010 & 2011 & 2012 \\
\hline \multirow{2}{*}{$\begin{array}{l}\text { Tasa de acceso neta, } \\
\text { inicial (\% estudiantes } \\
\text { de } 3 \text { a } 5 \text { años) }\end{array}$} & Perú & 65.6 & 67.6 & 67.8 & 70.3 & 72.6 & 74.6 \\
\hline & Madre de Dios & 64.4 & 66.9 & 60.7 & 74.3 & 60.9 & 72.7 \\
\hline \multirow{2}{*}{$\begin{array}{l}\text { Tasa de acceso neta, } \\
\text { primaria (\% estudi- } \\
\text { antes de } 6 \text { a } 11 \text { años) }\end{array}$} & Perú & 93.6 & 94.2 & 94.3 & 94.0 & 94.0 & 92.9 \\
\hline & Madre de Dios & 95.9 & 97.4 & 95.1 & 96.0 & 93.4 & 93.3 \\
\hline \multirow{2}{*}{$\begin{array}{l}\text { Tasa de acceso neta, } \\
\text { secundaria (\% estudi- } \\
\text { antes de } 12 \text { a } 16 \text { años) }\end{array}$} & Perú & 76.0 & 76.2 & 77.8 & 79.2 & 80.0 & 80.7 \\
\hline & Madre de Dios & 81.5 & 83.1 & 84.0 & 83.4 & 82.3 & 81.7 \\
\hline \multirow{2}{*}{$\begin{array}{l}\text { Tasa de acceso bruta, } \\
\text { superior (\% estudi- } \\
\text { antes de } 17 \text { a } 21 \text { años) }\end{array}$} & Perú & 51.6 & 53.4 & 55.0 & 57.1 & 62.1 & 69.1 \\
\hline & Madre de Dios & 46.8 & 59.3 & 64.2 & 76.7 & 64.3 & 77.4 \\
\hline
\end{tabular}

Fuente: Ministerio de Educación, Secretaria de Planificación Estratégica,

Oficina de Seguimiento y Evaluación Estratégica, Unidad de Estadística, 2013

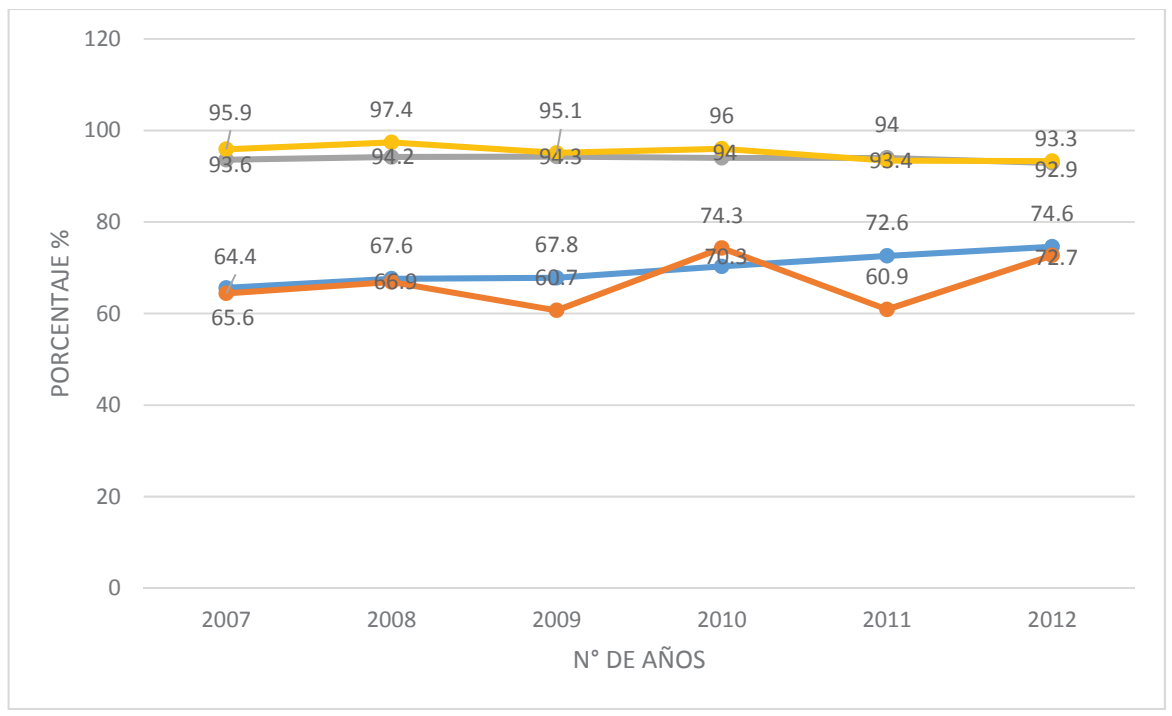

\section{Gráfico $N^{\circ}$ 02. Acceso Educativo en Inicial y Primaria}

Fuente: Ministerio de Educación, Secretaría de Planificación Estratégica, Oficina de Seguimiento y Evaluación Estratégica, Unidad de Estadística, 2013. 


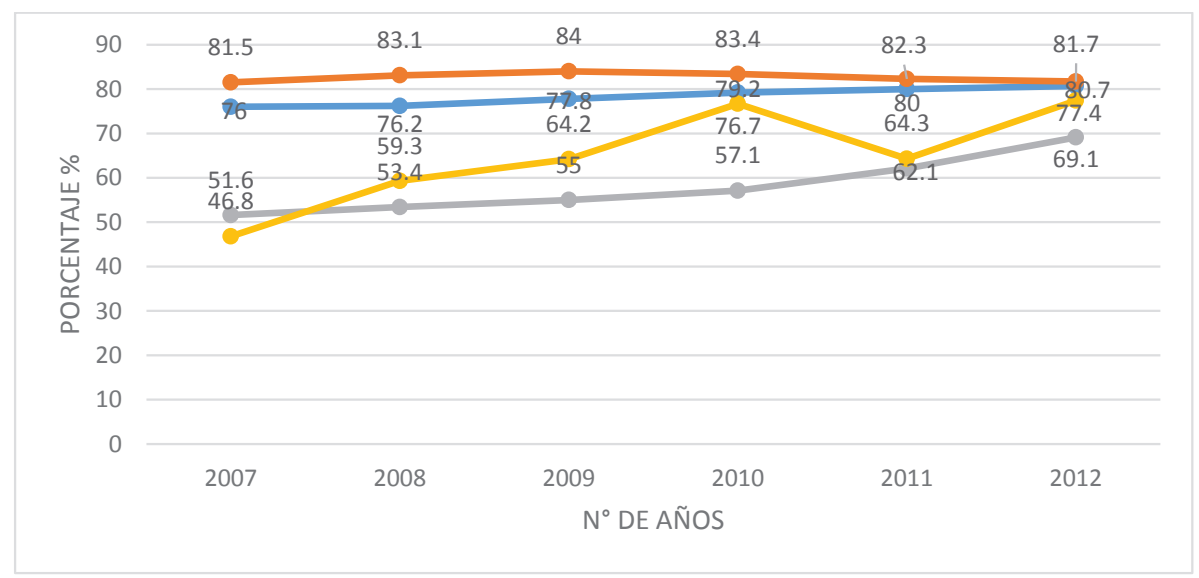

Gráfico $\mathbf{N}^{\circ}$ 03. Acceso Educativo en Secundaria y Superior

Fuente: Fuente: Ministerio de Educación, Secretaría de Planificación Estratégica, Oficina de Seguimiento y Evaluación Estratégica, Unidad de Estadística, 2013.

\section{Indicadores de conclusión educativa}

En Madre de Dios, los indicadores de conclusión educativa en el 2013 indican que el 93.1\% de las y los estudiantes de 12 a 14 años terminó primaria, y el grupo etáreo de 15 a 19 concluyo el 97.4\%. En secundaria hubo un crecimiento en los indicadores en el grupo etáreo de 17-18 con 64.9\%, en el de 17-19 fué $69.6 .5 \%$ y en el grupo de $20-24$ fué $82.7 \%$. En superior en los rangos de $22-24$ años $18.4 \%$, de $25-34$ años $25.4 \%$.

Cuadro $\mathbf{N}^{\circ}$ 07. Indicadores de Conclusión Primaria

\begin{tabular}{lcccccc} 
& \multicolumn{3}{c}{ Grupo de edades - años } \\
Ámbito & \multicolumn{2}{c}{$\mathbf{1 2 - 1 3}$} & \multicolumn{2}{c}{$\mathbf{1 2 - \mathbf { 1 4 }}$} & \multicolumn{2}{c}{$\mathbf{1 5} \mathbf{- 1 9}$} \\
& $\mathbf{2 0 0 7}$ & $\mathbf{2 0 1 3}$ & $\mathbf{2 0 0 7}$ & $\mathbf{2 0 1 3}$ & $\mathbf{2 0 0 7}$ & $\mathbf{2 0 1 3}$ \\
Perú & 74.1 & 80.3 & 79.2 & 84.8 & 94.3 & 96.9 \\
Madre de Dios & 79.4 & 92 & 84.8 & 93.1 & 98.1 & 97.4 \\
\hline
\end{tabular}

Fuente: Ministerio de Educación, Secretaría de Planificación Estratégica, Oficina de Seguimiento y Evaluación Estratégica, Unidad de Estadística, 2014 
Cuadro $\mathbf{N}^{\circ}$ 08. Indicadores de Conclusión Secundaria

\begin{tabular}{lcccccc} 
& \multicolumn{4}{c}{ Grupo de edades - años } \\
Ámbito & \multicolumn{2}{c}{$\mathbf{1 7 - 1 8}$} & \multicolumn{2}{c}{$\mathbf{1 7 - 1 9}$} & \multicolumn{2}{c}{$\mathbf{2 0 - 2 4}$} \\
& $\mathbf{2 0 0 7}$ & $\mathbf{2 0 1 3}$ & $\mathbf{2 0 0 7}$ & $\mathbf{2 0 1 3}$ & $\mathbf{2 0 0 7}$ & $\mathbf{2 0 1 3}$ \\
Perú & 57.6 & 64.8 & 61.5 & 69.2 & 72.4 & 82 \\
Madre de Dios & 59.7 & 64.9 & 63.8 & 69.6 & 70 & 82.7 \\
\hline
\end{tabular}

Fuente: Ministerio de Educación, Secretaría de Planificación Estratégica, Oficina de Seguimiento y Evaluación, Unidad de Estadística, 2014.

Cuadro $\mathbf{N}^{\circ}$ 09. Indicadores de Conclusión Educación Superior

\begin{tabular}{lcccc} 
& \multicolumn{5}{c}{ Grupo de edades - años } \\
Ámbito & $\mathbf{2 2 - 2 4}$ & & $\mathbf{2 5 - 3 4}$ \\
& $\mathbf{2 0 0 7}$ & $\mathbf{2 0 1 3}$ & $\mathbf{2 0 0 7}$ & $\mathbf{2 0 1 3}$ \\
Perú & 15.3 & 19.8 & 23.2 & 27.4 \\
Madre de Dios & 8.8 & 18.4 & 17.8 & 25.4 \\
\hline
\end{tabular}

Fuente: Ministerio de Educación, Secretaría de Planificación Estratégica, Oficina de Seguimiento y Evaluación Estratégica, Unidad de Estadística, 2014.

\section{Indicadores de Nivel educativo concluido}

El 2013, el 47.3\% de la población completo la Secundaria, el 12.3\% culminó formación superior no universitaria, el 11.5\% concluyó superior universitaria y el 1.5\% finalizó estudios de post-grado.

Cuadro $\mathbf{N}^{\circ}$ 10. Población de 25-34 años por Nivel Educativo Concluido

\begin{tabular}{lllllllllllllll} 
& $\begin{array}{c}\text { Primaria } \\
\text { incompleta o } \\
\text { Ámbito }\end{array}$ & \multicolumn{2}{c}{$\begin{array}{c}\text { Primaria } \\
\text { completa }\end{array}$} & \multicolumn{2}{c}{$\begin{array}{c}\text { Secundaria } \\
\text { completa }\end{array}$} & $\begin{array}{c}\text { Superior no } \\
\text { universitaria } \\
\text { completa }\end{array}$ & $\begin{array}{c}\text { Superior } \\
\text { universitaria } \\
\text { completa }\end{array}$ & Post-Grado \\
& $\mathbf{2 0 1 2}$ & $\mathbf{2 0 1 3}$ & $\mathbf{2 0 1 2}$ & $\mathbf{2 0 1 3}$ & $\mathbf{2 0 1 2}$ & $\mathbf{2 0 1 3}$ & $\mathbf{2 0 1 2}$ & $\mathbf{2 0 1 3}$ & $\mathbf{2 0 1 2}$ & $\mathbf{2 0 1 3}$ & $\mathbf{2 0 1 2}$ & $\mathbf{2 0 1 3}$ \\
Perú & 8.7 & 8.5 & 19.7 & 19.5 & 44.9 & 44.6 & 13.4 & 13.5 & 11.6 & 12.2 & 1.8 & 1.6 \\
$\begin{array}{l}\text { Madre de } \\
\text { Dios }\end{array}$ & 7.2 & 4.2 & 24.7 & 23.1 & 46.1 & 47.3 & 12.9 & 12.3 & 8.8 & 11.5 & 0.3 & 1.5 \\
\hline
\end{tabular}

Fuente: Ministerio de Educación, Secretaría de Planificación Estratégica, Oficina de Seguimiento y Evaluación Estratégica, Unidad de Estadística, 2014.

Los años de estudios de la población adulta para el año 2013, fue de 10.0 años a nivel nacional, 9.8 años para Madre de Dios cercano al promedio nacional. 
Cuadro $\mathbf{N}^{\circ}$ 11. Años de Estudios de la Población Adulta

\begin{tabular}{lccccc} 
& \multicolumn{5}{c}{ Grupo de edades - años } \\
Ámbito & $\mathbf{2 5 - 3 4}$ & & $\mathbf{2 5} \mathbf{- 6 4}$ & \\
& $\mathbf{2 0 1 2}$ & $\mathbf{2 0 1 3}$ & $\mathbf{2 0 1 2}$ & $\mathbf{2 0 1 3}$ \\
Perú & 11.1 & 11.2 & 9.9 & 10 \\
Madre de Dios & 11 & 11.5 & 9.8 & 9.8 \\
\hline
\end{tabular}

Fuente: Ministerio de Educación, Secretaría de Planificación Estratégica, Oficina de Seguimiento y Evaluación Estratégica, Unidad de Estadística, 2014.

\section{Resultado y Discusión}

\section{a. Diseño de Comprobación de la Investigación}

La comprobación de nuestra investigación, que argumenta la importancia de las inversiones en desarrollo humano, así como las estrategias educativas y la disponibilidad de profesionales docentes y no docentes de alta especialización, en el crecimiento y desarrollo económico de Madre de Dios, ha sido comprobado a través de los resultados obtenidos de las encuestas y entrevistas realizadas a personas involucradas en el tema, con residencia en el referido departamento. Principalmente, jóvenes universitarios, profesionales independientes y docentes.

\section{i. Determinación del Número de Entrevistas y Aplicación de Encuestas}

Es preciso remarcar de antemano que para determinar el tamaño de nuestra muestra se ha aplicado una formula estadística, donde tomaremos en cuenta el parámetro a estimar, la precisión y el nivel de confianza (1 - @), que habitualmente es el 95\% o 99\%.

Como nuestra población es infinita, toda vez que muchas son las personas relacionadas con este sector, sé aplicado la siguiente formula:

\section{ii. Fórmula}

Para determinar la cantidad de entrevistas a realizar se aplica la fórmula del tamaño muestral, con una probabilidad de certeza en los resultados de 1.96, de acuerdo al a tabla de Gauss y con un error muestral de10\%. 


$$
n=Z \frac{N * p * q}{I(N-1)+Z p * q}
$$

\section{iii. Donde:}

Para efectos de obtener la cantidad de entrevistas a realizar, tomaremos como tamaño muestral, el 50\% de nuestra población real $(2,000$ personas entre universitarios, profesionales independientes y docentes universitarios y no universitarios).

$\mathrm{n}=$ Tamaño muestral

$\mathrm{N}$ = Tamaño de la población

$\mathrm{Z}=$ Valor correspondiente a la distribución de Gauss 1.96 para $\alpha=$ 0.05 y 2.58 para $\alpha=0,01$.

$\mathrm{p}=$ Prevalencia esperada del parámetro a evaluar.

$q=1-p($ Si $p=30 \%, q=70 \%)$

$\mathrm{i}=$ Error que se prevé cometer. Por ejemplo un error de $10 \%$ que en la formula es igual a $0.1 \%$

iv. Reemplazando Datos en la Fórmula

$$
\begin{gathered}
n=(1.96) 2 \frac{2000 * 0.85 * 0.15}{(0.1) 1 *(2000-1)+(1.96 * 0.85 * 0.15)} \\
n=3.8416 \frac{255}{(0.01)(1999)+(0.25)} \\
n=3.8416 \frac{255}{19.99+0.25} \\
n=3.8416 \frac{255}{20.24} \\
n=3.8416(12.60)=48.40 \%
\end{gathered}
$$


Para ejecutar la entrevista tendríamos que ejecutar 968 entrevistas en nuestra población objetivo, como resultado de aplicar el $48.40 \%$ de 2000 personas.

\section{b. Encuesta}

Como mencionáramos anteriormente, los resultados obtenidos son producto de la aplicación de un test de encuestas a las personas directamente relacionadas con el crecimiento y desarrollo económico del departamento de Madre de Dios, desde una perspectiva del capital humano.

Las preguntas realizadas en nuestras entrevistas son las que a continuación se detallan:

1. Considera Ud. que el crecimiento y desarrollo económico del departamento de Madre de Dios, esta ligado significativamente al desarrollo de su capital humano?

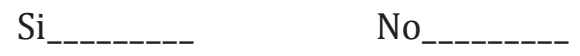

2. Cómo calificaría Ud. el actual desarrollo humano en el departamento de Madre de Dios?

Es pobre

Medianamente Pobre

Medianamente competitivo

Muy competitivo

3. Considera usted, que se vienen aplicando las correctas estrategias educativas, para que el departamento logre alcanzar un desarrollo humano competitivo que a su vez redunde en un crecimiento y desarrollo económico del mismo?

No existen estrategias educativas

Las estrategias aplicadas son deficientes

Las estrategias educativas son eficientes 
4. Los profesionales docentes y no docentes de Madre de Dios, cuentan con alta especialización de calidad que contribuyen al desarrollo humano y a la vez al crecimiento y desarrollo económico del departamento?

Si son competitivos

No son competitivos

5. A su consideración, cree Ud. que el gobierno Local, Regional y Nacional, trabajan coordinadamente para lograr una educación competitiva en el departamento de Madre de Dios?

Si trabajan coordinadamente

No trabajan coordinadamente

6. Existe actualmente inversión pública y privada en educación, que permita alcanzar un mayor desarrollo humano en el departamento de Madre de Dios?

Si existe

No existe

7. Considera Ud. que la capacitación de profesionales docentes y no docentes es:

Muy Importante

Importante

Poco Importante

Nada Importante

8. Cual cree Ud. que es la mejor alternativa de desarrollo humano en Madre de Dios, la proveniente de:

El Sector Privado

El Sector Público 


\section{Ambos Sectores}

9. De que forma podría mejorar la relación Inversión - Educación Desarrollo Humano - Crecimiento y Desarrollo Económico

Alianzas Estratégicas público- privadas

Mejores Políticas Gubernamentales

Otros

\section{i. Cuadro de Resultados}

Cuadro $\mathbf{N}^{\circ} \mathbf{1 2}$. De resultados obtenidos

\begin{tabular}{|c|c|c|c|}
\hline PREGUNTA & CONDICIONES PREGUNTAS & CANTIDAD ENTREVISTAS & PORCENTAJE \% \\
\hline \multirow{2}{*}{1} & $\mathrm{Si}$ & 785 & 81.10 \\
\hline & No & 183 & 18.90 \\
\hline \multirow{4}{*}{2} & Es pobre & 459 & 47.42 \\
\hline & Medianamente Pobre & 283 & 29.24 \\
\hline & Medianamente competitivo & 151 & 15.60 \\
\hline & Muy competitivo & 75 & 7.74 \\
\hline \multirow{3}{*}{3} & No existen estrategias educativas. & 497 & 51.34 \\
\hline & Las estrategias aplicadas son deficientes. & 358 & 36.98 \\
\hline & Las estrategias educativas son eficientes. & 113 & 11.68 \\
\hline \multirow{2}{*}{4} & Si son competitivos & 822 & 84.92 \\
\hline & No son competitivos & 146 & 15.08 \\
\hline \multirow{2}{*}{5} & Si trabajan coordinadamente & 452 & 46.69 \\
\hline & No trabajan coordinadamente & 516 & 53.31 \\
\hline \multirow{2}{*}{6} & Si existe & 884 & 91.32 \\
\hline & No existe & 84 & 8.68 \\
\hline \multirow{4}{*}{7} & Muy Importante & & \\
\hline & Importante & 785 & 81.10 \\
\hline & Poco Importante & 183 & 18.90 \\
\hline & Nada Importante & & \\
\hline \multirow{3}{*}{8} & Sector Privado & 332 & 34.30 \\
\hline & Sector Público & 455 & 47.00 \\
\hline & Ambos sectores & 181 & 18.70 \\
\hline \multirow{3}{*}{9} & Alianzas Estratégicas & 551 & 56.92 \\
\hline & Políticas Gubernamentales & 352 & 36.36 \\
\hline & Otros & 65 & 6.72 \\
\hline
\end{tabular}

Fuente: Cuestionario de Entrevista / Elaboración propia 


\section{ii. Análisis de los Resultados Obtenidos}

Como mencionáramos anteriormente, los resultados obtenidos son producto de la aplicación de un test de encuestas realizadas a un grupo de personas relacionadas con el sector educativo de Madre de Dios, su desarrollo económico y que son objeto de nuestro estudio.

\section{Análisis de Encuesta}

1. Considera Ud. que el crecimiento y desarrollo económico del departamento de Madre de Dios, esta ligado significativamente al desarrollo de su capital humano?

\begin{tabular}{|c|c|c|}
\multicolumn{3}{|c}{ No } \\
\hline ALTERNATIVAS & $\begin{array}{c}\text { CANTIDAD DE } \\
\text { ENTREVISTADOS }\end{array}$ & PORCENTAJE \\
\hline SI & 785 & 81.10 \\
\hline NO & 163 & 18.90 \\
\hline
\end{tabular}

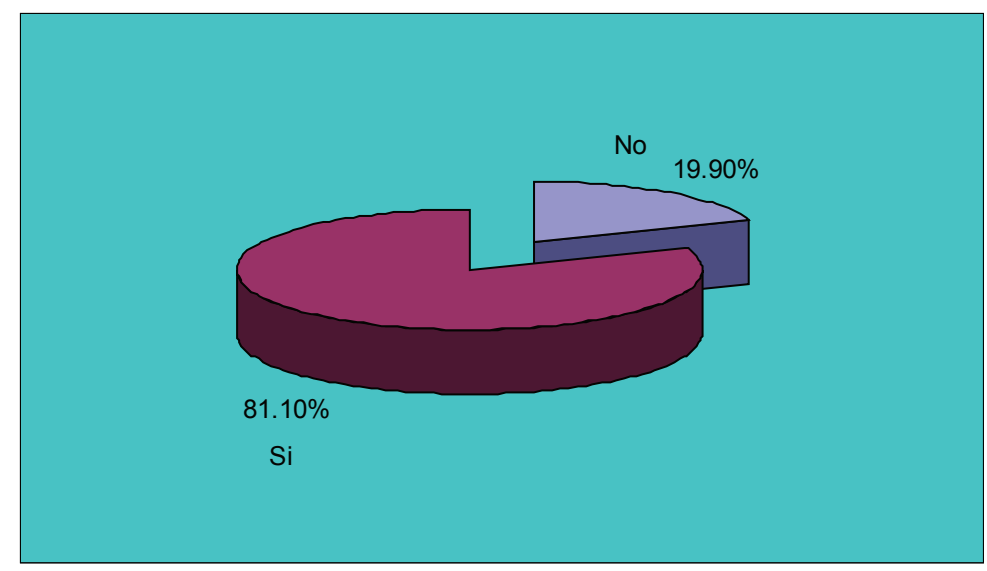

Según los resultados obtenidos en las entrevistas realizadas, el $81.10 \%$ de los encuestados, manifestaron que existe una relación significativa entre el crecimiento y desarrollo económico de Madre de Dios y el desarrollo del capital humano. 
2. Cómo calificaría Ud. el actual desarrollo humano en el departamento de Madre de Dios?

Es pobre

Medianamente Pobre

Medianamente competitivo

Muy competitivo

\begin{tabular}{|l|c|c|}
\hline ALTERNATIVAS & $\begin{array}{c}\text { CANTIDAD DE } \\
\text { ENTREVISTADOS }\end{array}$ & PORCENTAJE \\
\hline Es pobre & 459 & 47.42 \\
\hline Medianamente Pobre & 283 & 29.24 \\
\hline Medianamente competitivo & 151 & 15.60 \\
\hline Muy competitivo & 75 & 7.74 \\
\hline
\end{tabular}

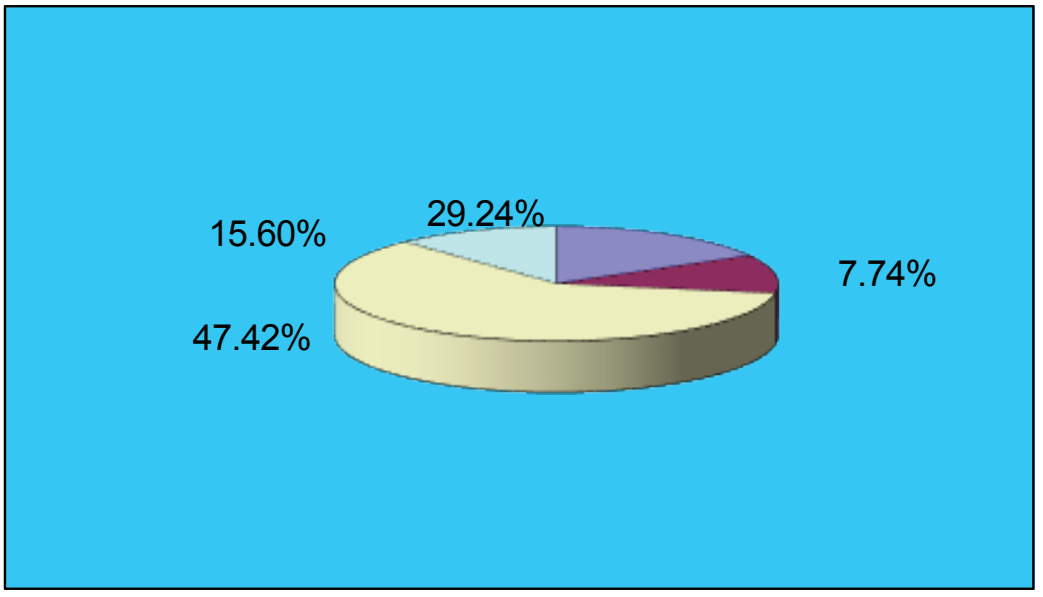

Según los resultados obtenidos, el $47.42 \%$ considera que el nivel de desarrollo humano en el departamento es pobre, un porcentaje significativo, si lo comparamos con el $29.24 \%$ que indica que es medianamente pobre, un $15.60 \%$ y $7.74 \%$ que indica que son medianamente competitivo y muy competitivo. 
3. Considera usted, que se vienen aplicando las correctas estrategias educativas, para que el departamento logre alcanzar un desarrollo humano competitivo que a su vez redunde en un crecimiento y desarrollo económico del mismo?

No existen estrategias educativas

Las estrategias aplicadas son deficientes

Las estrategias educativas son eficientes

\begin{tabular}{|l|c|c|}
\hline ALTERNATIVAS & $\begin{array}{c}\text { CANTIDAD DE } \\
\text { ENTREVISTADOS }\end{array}$ & PORCENTAJE \\
\hline No existen estrategias educativas & 497 & 51.34 \\
\hline Las estrategias aplicadas son deficientes & 358 & 36.98 \\
\hline Las estrategias educativas son eficientes & 113 & 11.68 \\
\hline
\end{tabular}

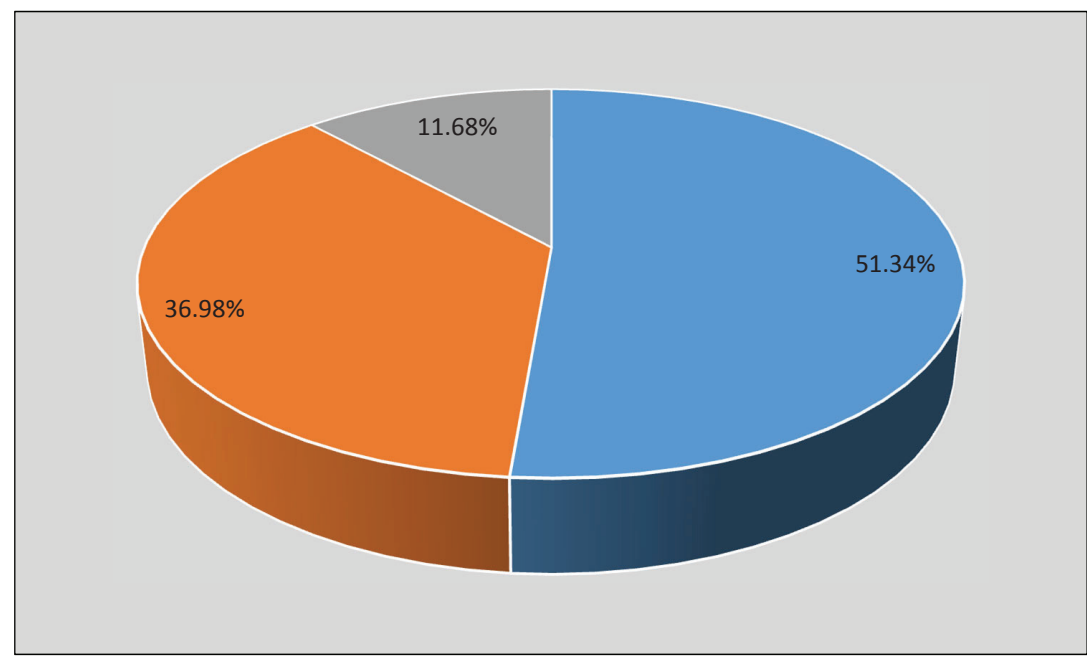

Con relación a la aplicación adecuada de estrategias educativas, el $51.34 \%$ manifestó que no existen estrategias educativas, frente a un $36.98 \%$ que indican que si existe pero éstas son eficientes y $11.68 \%$ que indica lo contrario, es decir son eficientes. 
4. Los profesionales docentes y no docentes de Madre de Dios, cuentan con alta especialización de calidad que contribuyen al desarrollo humano y a la vez al crecimiento y desarrollo económico del departamento?

Si son competitivos

No son competitivos

\begin{tabular}{|l|c|c|}
\hline \multicolumn{1}{|c|}{ ALTERNATIVAS } & \multicolumn{1}{c|}{$\begin{array}{c}\text { CANTIDAD DE } \\
\text { ENTREVISTADOS }\end{array}$} & \multicolumn{1}{c|}{ PORCENTAJE } \\
\hline Si son competitivos & 822 & 84.92 \\
\hline No son competitivos & 146 & 15.08 \\
\hline
\end{tabular}

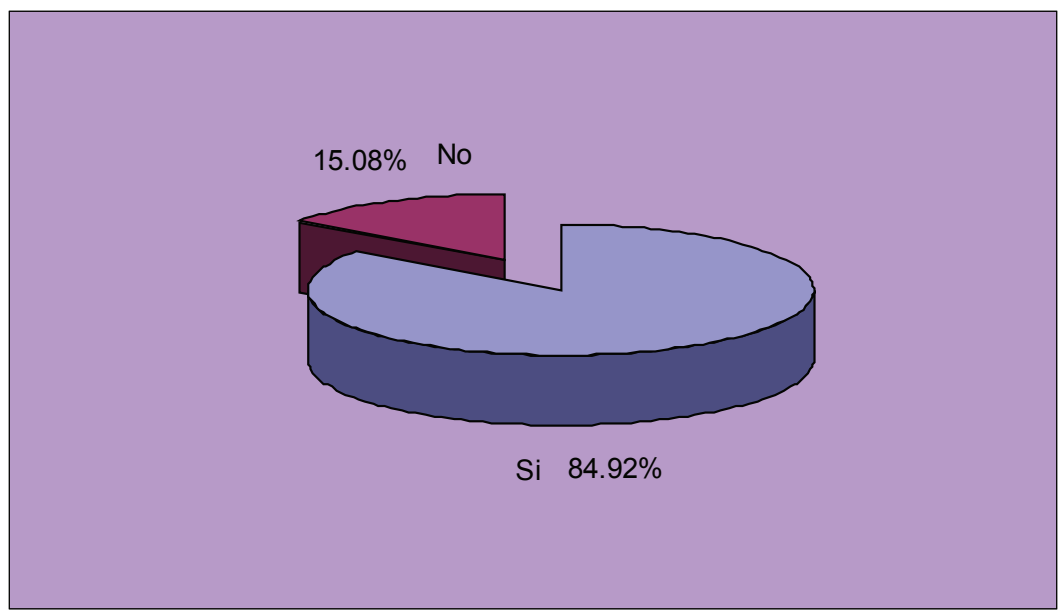

Datos obtenidos de la encuesta efectuada, indica que el $84.92 \%$ de los entrevistados, considera que los profesionales docentes y no docentes son competitivos en el departamento de Madre de Dios, frente al $15.08 \%$ que indica lo contrario. 
5. A su consideración, cree Ud. que el gobierno Local, Regional y Nacional, trabajan coordinadamente para lograr una educación competitiva en el departamento de Madre de Dios?

Si trabajan coordinadamente

No trabajan coordinadamente

\begin{tabular}{|l|c|c|}
\hline ALTERNATIVAS & $\begin{array}{c}\text { CANTIDAD DE } \\
\text { ENTREVISTADOS }\end{array}$ & PORCENTAJE \\
\hline Si trabajan coordinadamente & 452 & 46.69 \\
\hline No trabajan coordinadamente & 516 & 53.31 \\
\hline
\end{tabular}

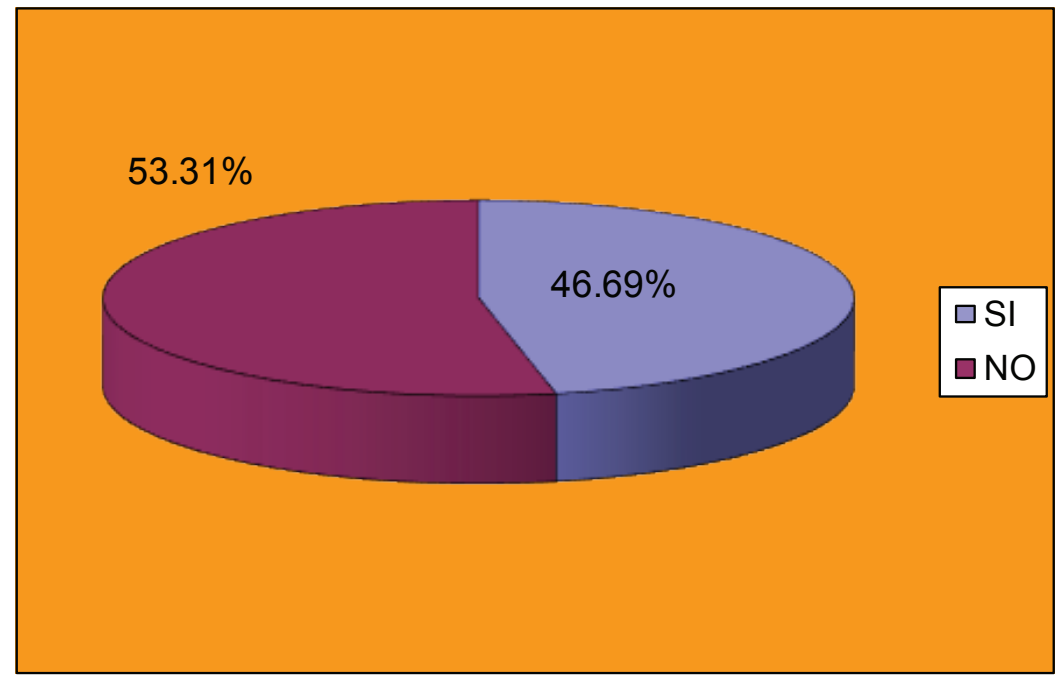

Ante esta pregunta, el 46.69\% manifestó que si existe coordinación entre los tres niveles de gobierno en cuanto a temas educativos, lo cual no es compartido por el $53.31 \%$ de los entrevistados que indicaron lo contrario. 
6. Existe actualmente inversión pública y privada en educación, que permita alcanzar un mayor desarrollo humano en el departamento de Madre de Dios?

Si existe

No existe

\begin{tabular}{|l|c|c|}
\hline ALTERNATIVAS & $\begin{array}{c}\text { CANTIDAD DE } \\
\text { ENTREVISTADOS }\end{array}$ & PORCENTAJE \\
\hline Si existe & 884 & 91.32 \\
\hline No existe & 84 & 8.68 \\
\hline
\end{tabular}

$8.68 \%$ No existe

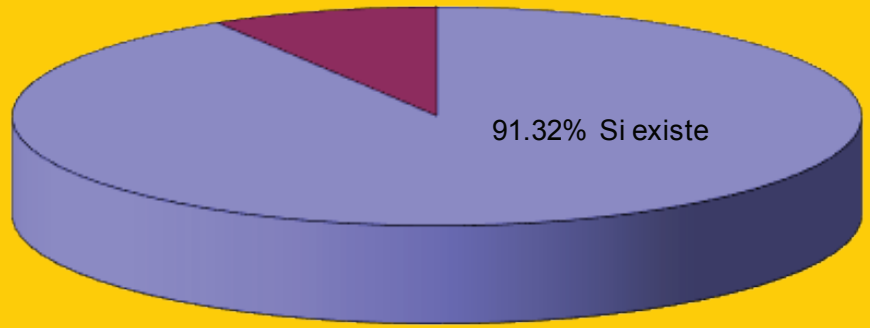

El 91.32\% manifestó que si se viene efectuando inversión en al ámbito educativo, en el departamento, aunque no se pudo determinar de que sector proviene. Por el contrario el $8.68 \%$ indico que no existe inversión en este sector. 
7. Considera Ud. que la capacitación de profesionales docentes y no docentes es:

Muy Importante

Importante

Poco Importante

Nada Importante

\begin{tabular}{|l|c|c|}
\hline ALTERNATIVAS & $\begin{array}{c}\text { CANTIDAD DE } \\
\text { EMPRESAS }\end{array}$ & PORCENTAJE \\
\hline Muy Importante & 785 & 81.10 \\
\hline importante & 183 & 18.90 \\
\hline Poco importante & 0 & 00.00 \\
\hline Nada importante & 0 & 00.00 \\
\hline
\end{tabular}

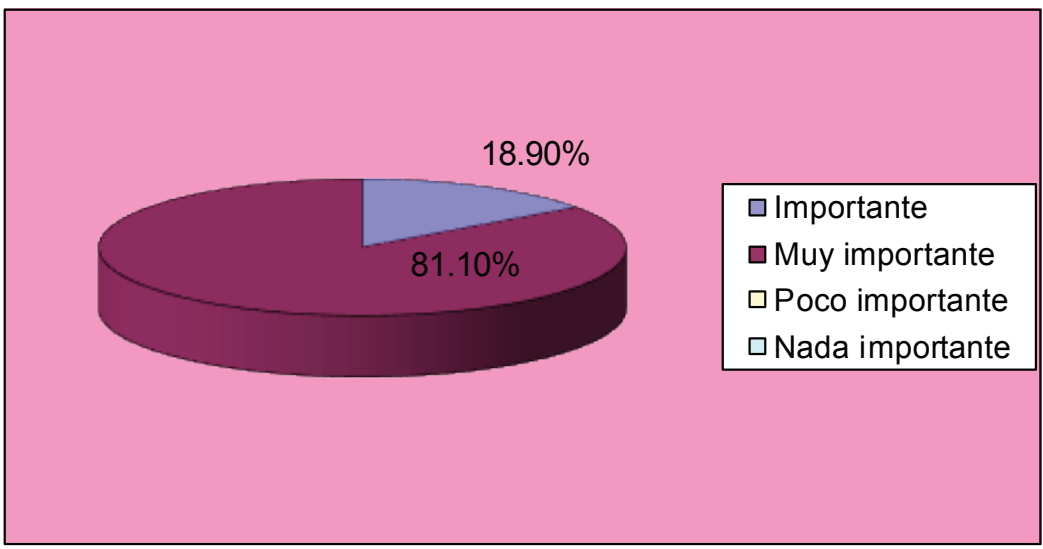

En cuanto a la importancia de la capacitación de profesionales docentes y no docentes, existe una fuerte opinión respecto a la importancia de ésta, que se refleja en el $81.10 \%$ de entrevistados, que contestó que era muy importante y un $18.90 \%$ que indico que era importante. En este caso no se admite, que la capacitación profesional, sea poco o nada importante. 
8. Cual cree Ud. que es la mejor alternativa de desarrollo humano en Madre de Dios, la proveniente de:

El Sector Privado

El Sector Público

Ambos Sectores

\begin{tabular}{|l|c|c|}
\hline ALTERNATIVAS & $\begin{array}{c}\text { CANTIDAD DE } \\
\text { ENTREVISTADOS }\end{array}$ & PORCENTAJE \\
\hline Sector Privado & 332 & 34.30 \\
\hline Sector Público & 455 & 47.00 \\
\hline Ambos Sectores & 181 & 18.70 \\
\hline
\end{tabular}

$18.70 \%$ Sector Privado

$34.30 \%$ Ambos Sectores

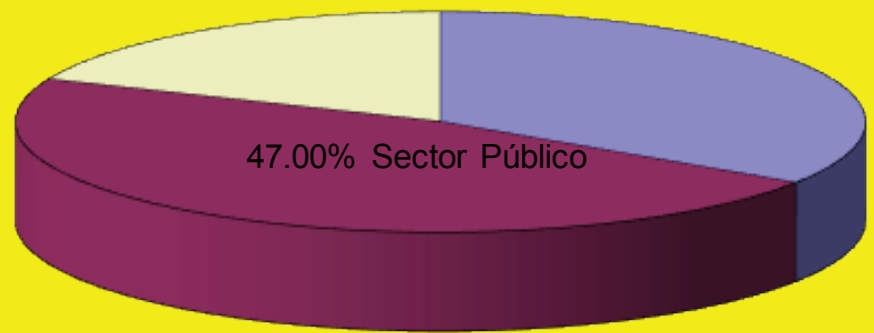

Ante esta pregunta, un $47 \%$ indica que el desarrollo humano en la región, debe provenir principalmente del sector público, frente a un $34.30 \%$ que indica que debe provenir de una mayor acción del sector privado y un $18.70 \%$ que debe provenir del conjunto de ambos sectores. 
9. De que forma podría mejorar la relación Inversión - Educación Desarrollo Humano - Crecimiento y Desarrollo Económico

Alianzas Estratégicas público- privadas

Mejores Políticas Gubernamentales

Otros

\begin{tabular}{|l|c|c|}
\hline ALTERNATIVAS & $\begin{array}{c}\text { CANTIDAD DE } \\
\text { ENTREVISTADOS }\end{array}$ & PORCENTAJE \\
\hline Alianzas Estratégicas & 551 & 56.92 \\
\hline Mejores Políticas Gubernamentales & 352 & 36.36 \\
\hline Otros & 65 & 6.72 \\
\hline
\end{tabular}

$6.72 \%$ Otros

$36.36 \%$ Mejores Políticas

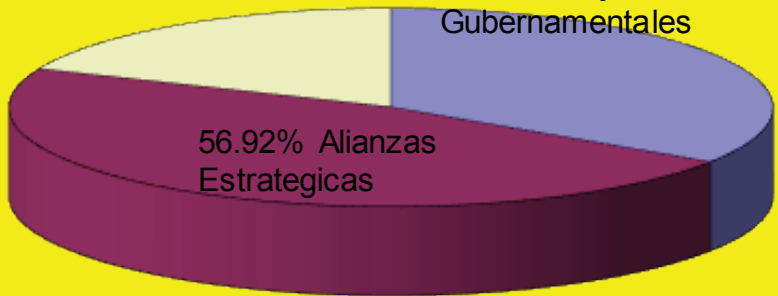

La mejor forma en que se puede mejorar esta relación, según el $56.92 \%$ de los entrevistados, es a través de alianzas estratégicas, frente al 36.36\% que indica que es a través de mejores políticas gubernamentales y un $6.72 \%$ que indica mediante otros mecanismos, sin formular cuales serían. 


\section{iii. Plan de Inversión en Educación desde un enfoque de Desar- rollo Humano en Madre de Dios.}

\section{Objetivo a Largo Plazo}

Asegurar la calidad de la educación en Madre de Dios con equidad, visión inclusiva, con enfoque de deberes y derechos para vigorizar la formación de su ciudadanía y cualificar el desarrollo humano y social contribuyendo a la reducción de la pobreza.

\section{Objetivos Estratégicos}

- Consolidar una reforma curricular en coordinación con el Ministerio de Educación concordado con la realidad de Madre de Dios.

- Renovar el plan de estudios del personal docente, actualizar al personal administrativo y de apoyo y contribuir al bienestar de esta población.

- Evaluar nuevas fuentes financieras que garanticen la sostenibilidad de la nueva estructura educativa.

- Promover una activa participación ciudadana

\section{Objetivos Específicos}

\section{Capital Humano}

Nuevo Plan de estudios docente Nueva Actualización y desarrollo profesional

Nuevo Acompañamiento, Monitoreo y Seguimiento Educativo Nueva Actualización del personal administrativo y de apoyo

\section{Visión}

Sistema educativo regional andino-amazónico integral y flexible, que satisface las necesidades priorizadas de aprendizaje social, y promueve nuestra identidad regional y las oportunidades educativas a nuestros niños, niñas, adolescentes, jóvenes, adultos y adultos mayores. 


\section{Misión}

Promover, a través de las instituciones educativas en su conjunto, una educación de calidad que procure el desarrollo humano como factor decisivo en el desarrollo integral y sostenible de Madre de Dios y satisfaga los requerimientos socio- educativos de la población.

\section{Estrategias del Nuevo Plan Regional de Inversión en Educación}

- Generar oportunidades para mejorar la calidad de vida de la población, contribuir a la reducción de las diferencias sociales.

- Promover el capital humano para incentivar la investigación y la innovación para generar tecnología que coadyube al crecimiento sostenible.

- Generar enseñanzas y aprendizajes en niños, niñas, adolescentes, jóvenes, adultos y adultos mayores para el ejercicio futuro de su ciudadanía en los espacios institucionales y organizacionales privados y públicos.

\section{Políticas Regionales}

Generalización de la educación inicial de 2 a 5 años de edad

Esta etapa de la vida del ser humano es la mejor para lograr una instrucción y educación optima ya que en este intervalo de tiempo se desarrolla la inteligencia y la identidad.

Universalización de la Educación Primaria

Para que niños y niñas de zona rural y urbano marginal incrementen habilidades que les estimulen aprender a convivir con los demás en su entorno social y natural, conociendo su identidad regional, respetando los derechos humanos y a la naturaleza.

Potenciar el nivel secundario

Preparar al alumnado, a seguir estudios superiores; capacitarlos para su incorporación a la producción, con calificación, y participen como ciudadanas/os, en el gobierno local, regional y nacional. 
Erradicar el Analfabetismo y Fortalecimiento de la Educación Alternativa

Formular programas a nivel regional en concordancia con los lineamientos educativos nacionales, que garanticen el acceso, permanencia, continuación y conclusión efectiva de los estudios de la población con rezago educativo.

Mejoramiento de la Infraestructura Física y equipamiento de los centros educativos

Considerando que el desarrollo humano requiere de diversos factores para su fortalecimiento, es importante y prioritaria la calidad de los servicios educativos, con recursos tecnológicos modernos que contribuyan al desarrollo de las/os estudiantes de Madre de Dios y al desarrollo integral de la región.

Implementar un Sistema Regional de Evaluación

Que permita ofrecer a los niños, niñas, adolescentes, jóvenes, adultos y adultos mayores, oportunidades, para disminuir las brechas sociales.

Puesta en valor de la Profesión Magisterial

Promocionar el libre acceso, promoción y condiciones de bienestar de los docentes.

Incremento Continuo del Presupuesto Regional a la Educación

Generar mayores recursos a la Dirección de Educación del Gobierno Regional, para mejorar la competitividad del mismo, a nivel nacional y que contribuya al desarrollo regional.

Implementar Programas de Formación Universitaria de Pregrado

- Ingeniería de Minas

- Ingeniería Geológica

- Ingeniería Metalúrgica 
Implementar Programa de Manejo Tecnológico en la Producción de Oro Aluvial.

Generar mayores recursos a la Dirección de Energía, Minas e Hidrocarburos del Gobierno Regional para aplicar el uso tecnológico moderno en la producción del Oro Aluvial y que contribuya al desarrollo regional.

\section{Conclusiones}

1. Según los resultados obtenidos en las 968 entrevistas realizadas, el $81.10 \%$ de los encuestados manifestaron que existe una relación significativa entre el crecimiento y el desarrollo económico con el desarrollo del capital humano.

2. Los entrevistados el $47.42 \%$ considera que el nivel de desarrollo humano en el departamento es pobre, un porcentaje significativo si lo comparamos con el $29.24 \%$ que indica que es medianamente pobre y el $15.60 \%$ indica que es medianamente competitivo y el $7.74 \%$ señala que es muy competitivo.

3. Con relación a la aplicación adecuada de estrategias educativas, el $51.34 \%$ manifestó que no existen estrategias educativas, un $36.98 \%$ indican que si existen pero estas son deficientes y un $11.68 \%$ indican que son eficientes.

4. Los datos obtenidos de las encuestas aplicadas indican que el $84.92 \%$ de los entrevistados consideran que los profesionales docentes y no docentes son competitivos y el $15.08 \%$ indican que no son competitivos.

5. Los entrevistados el $46.69 \%$ manifestaron que sí existe coordinación entre los tres niveles de gobierno en cuanto a la educación, lo que no es compartido por el $53.31 \%$ de los encuestados que indicaron que no trabajan coordinadamente.

6. De los encuestados el $91.32 \%$ manifestaron que se viene efectuando inversión pública y privada en el sector educativo y el 8.68\% indicó que no existe inversión en este ámbito. 
7. En cuanto a la importancia de la capacitación de profesionales docentes y no docentes, existe una fuerte opinión respecto a la importancia de esta que se refleja en el $81.10 \%$ de entrevistados, que manifestó que era muy importante y un $18.90 \%$ indico que era importante.

8. El $47 \%$ de los entrevistados señalan que el desarrollo humano en la región, debe provenir principalmente del sector público y el $34.30 \%$ indica que debe provenir de una mayor acción del sector privado y un $18.70 \%$ que debe provenir del conjunto de ambos sectores.

9. La encuesta de la presente investigación ha indicado que la población de la muestra considera que las alianzas público-privadas son las alternativas para mejorar la educación y el desarrollo humano con el $56.92 \%$ una cifra superior al $36.36 \%$ que espera una mejora en las políticas gubernamentales desde Lima.

\section{Recomendaciones}

1. Emplear la Beca 18 en las Universidades Privadas y Públicas del Departamento de Madre de Dios para formar Capital Humano apropiado a la Estrategia de Desarrollo Regional.

2. Impulsar Estrategias de Desarrollo Regional acordes a las potencialidades del Departamento en piscicultura, apicultura y avicultura.

3. Crear en la Universidad Nacional de Madre de Dios las siguientes profesiones vinculadas al potencial del Departamento:

- Ingeniería de Minas

- Ingeniería Geológica

- Ingeniería Metalúrgica.

4. Implementar en el Departamento un Programa de Manejo Tecnológico en la Producción de Oro Aluvial por la Dirección Regional de Energía, Minas e Hidrocarburos del Gobierno Regional. 
5. Crear la Maestría Internacional de Desarrollo Regional con doble titulación por convenio entre la Universidad Nacional Mayor de San Marcos y la Universidad Nacional de Madre de Dios para atraer a graduandos de países limítrofes.

6. La Alianza Gobierno Regional-Sector Privado podría darse en la Educación Técnica y Superior, esto significa la creación de Escuelas e Institutos Superiores de Educación Técnica y de una Universidad Privada con nuevas propuestas apropiadas a los recursos de Madre de Dios: Minería, Reforestación, Metalúrgia, Agroforestería y Turismo Ecológico.

7. El Gobierno Regional deberá convocar a los Organismos No Gubernamentales de Madre de Dios para ejecutar proyectos de Desarrollo Regional que ellos producen en sus estudios regionales, la convocatoria debería realizarse cada seis (06) meses. El modelo de convocar a las ONGs para ejecutar proyectos ha dado grandes frutos en la República de Chile.

8. Formar una Comisión Inter-gobiernos regionales de Economistas é Ingenieros a fin de transferir entre los departamentos las innovaciones y proyectos de éxito. Aprovechar y compartir el mejor Capital Humano actualmente existente entre sí.

9. El Gobierno Regional deberá tener activa participación en el Convenio Madre de Dios, Acre y Pando (MAP) con el fin de elaborar Programas de Desarrollo conjunto con el Estado de Acre-Brasil y el Departamento Autónomo de Pando-Bolivia, y efectuar pasantías e intercambio de experiencias a nivel de un trabajo conjunto en esta zona de frontera.

\section{Referencias Bibliográficas}

AGUAYO L. Eva. PORTILLO V. Saskia. EXPOSITOR D. Pilar

"Perspectivas del crecimiento económico en los países del PACTO Andina"

Working Paper $N^{\circ}$ 39, 12 Págs. 2003

Universidad de Santiago de Compostela, Sección de Económetra.

España 
http://www.use.es/econonomet/aea.html

ANDERSEN LYKKE. E.

"Educacion en Bolivia: El efecto sobre el crecimiento, el empleo, la desigualdad y la pobreza"

Instituto de Investigación de Investigaciones Socio - Económicos

Universidad Catolica de Bolivia. 2003

Bolivia. La Paz.

http://www.iisec.ueb.edu.bo/poeb/papers/

ARANCIBA, JUAN

"Informe preliminar sobre la situación de la educación en América Latina para la IEAL"

V conferencia Regional de la Internacional de la Educación

Costa Rica, San José. 2003

DARVILLE, PAULA; DIAZ, RODRIGO

"Financiamiento de la Educación: Investigación y Rendimientos"

Ministerio de Educación, Departamento de Estudios y Estadísticas, 18 Págs.

Chile, Santiago, 2003

DU BOIS, FRITZ

"Un balance de las Políticas Sociales"

Instituto Peruano de Economía Social de mercado

Primera Edición, Págs. 165, 2004

Perú, Lima

ESCOBAR, JAVIER Y SAAVEDRA, JAIME

"Los Activos de los Pobres en el Perú"

Banco Interamericano de Desarrollo (BID) - GRADE

Documentos de trabajo 26, Primera Edición.

Perú, Lima, 1999; 70 Págs.

FLORES CRESPO, PEDRO

“¿Puede la educación generar desarrollo?” 
Revista Electrónica de Investigación Educativa, Vol 6, º 2, 6 págs.

Instituto de Investigaciones para el Desarrollo de la Educación

Universidad Iberoamericana

México, DF México, 2004, 6 págs.

COHEN, Ernesto. (2000). Hacia dónde va el gasto público en educación? Logros y desafíos. Vol. I. Serie Políticas Sociales, 42, Naciones Unidas, CEPAL, Santiago de Chile.

ARMIJO, MARIANELA (2003), La evaluación de la gestión pública en Chile. En Cunill Grau, Nuria y Ospina Bozzi, Sonia (comps.): Evaluación de Resultados para una Gestión Pública Moderna y Democrática: Experiencias latinoamericanas. CLAD; AECI/MAP/FIIAPP, Caracas.

CHUMBITA (1997) Evaluación en la gestión pública: Caso del Sistema Nacional de Evaluación Educativa. Instituto Nacional de la Administración Pública. Dirección Nacional de Estudios y Documentación, Dirección de Estudios e Investigación, Serie I. Desarrollo Institucional y Reforma del Estado, Buenos Aires.

HERNÁNDEZ SAMPIERI, Roberto y Otros. (2006). Metodología y Taller de Investigación. Editorial Mc. Graw Hill. México.

DIRECCION REGIONAL DE EDUCACION DE MADRE DE DIOS (2008) Plan Estratégico Institucional 2008-2011.

OROVAL PLANAS, Esteve. (1996). Economía de la Educación. Ariel. Barcelona. España.

MORÁN, Alberto; ZELLER, Norberto; RIVKIN, Ana; y LÓPEZ, Andrea (2003) Evolución de la Estructura Organizativa, Presupuesto y Personal de la Jefatura de Gabinete de Ministros, 1995 - 2002. Instituto Nacional de la Administración Pública. Dirección de Estudios e información, Buenos Aires.

LASSIBILLE, Gerard; NAVARRO GÓMEZ, Lucía (2012) Un compendio de investigaciones en economía de la educación. Presupuesto y Gasto Público 67/2012; Secretaria de Estado de Presupuesto y Gasto, Instituto de Estudios Fiscales Andalucía, España.

VEXLER TALLEDO, Idel (2004) Informe sobre la Educación Peruana Situación y perspectivas. CEPAL; Financiamiento y Gestión de la Educación en América Latina y el Caribe. 
ALCÁZAR, Lorena; VALDIVIA, Néstor (2011) Descentralización y Presupuesto de la Educación Pública en el Perú: Avances y Desafíos; Revista Peruana de Investigación Educativa № 2

WONG TORRES, Zelma; SALCEDO GUZMAN, Luisa Elena (2009) Factores clave para el Desarrollo y la estabilidad Económica en el Perú.

QUIPUKAMAYOC, Revista de la Facultad de Ciencias Contables, UNMSM.

MINISTERIO DE EDUCACIÓN. (2006) Perspectivas de la descentralización educativa en el Perú, 2006. Lima: Oficina de Coordinación y Supervisión Regional.

LASSIBILLE, Gerard y NAVARRO GOMEZ, M. Lucía (2004). Manual de Economía de la Educación: Teoría y Casos Prácticos Ediciones Pirámide. Madrid.

BRUNNER, José, ELACQUA, Gregory (2003) Informe Capital Humano en Chile Universidad Adolfo Ibáñez, Escuela de Gobierno, Chile, Santiago 